\title{
Masters of Forest Conservation Special Papers
}

W ith the publication of this select group of papers from its most recent graduating class, the Masters of Forest Conservation (MFC) Program at the University of Toronto's Faculty of Forestry joins the Canadian Institute of Forestry/Institut forestier du Canada to celebrate the successful completion of its $15^{\text {th }}$ year. Since 1996 when the program began, it has experienced considerable success, now totalling over 200 graduates, many of whom have gone on to work in the forest and natural resource sectors across Canada and throughout the world. The MFC program was established in recognition of fundamental values, focus and priorities desired and expected by the Canadian public, and to better address the much broader and truly interdisciplinary nature of forestry, forest science and natural resource management and conservation. The MFC program was designed to provide an intensive, 16-month course-based professional training in forest conservation, including practical field and laboratory experience.

The opportunity to feature some of our students' outstanding work through publication in The Forestry Chronicle is much appreciated, and something I hope will be built upon, and inspire other faculties across Canada to do the same. Our professors are very proud of the research that our new forest professionals undertake in the course of their program, and more specifically through the selective work in their major papers. Throughout the 12 months of project work and associated major paper, each student is supported by a team of both internal and external advisors, the latter helping to assure relevance and practical application within the forest and natural resources sector.

This year we were fortunate to have quite a number of outside individuals attend the paper presentations in December, including John Pineau, Executive Director of the CIF/IFC and Matt Meade, Forestry Extension Manager with the CIF/IFC and the Forestry Research Partnership (FRP), and a successful graduate of the MFC program. Based on a number of criteria including the presentation itself, writing style, topic, theme, scientific rigour, results and relevant information and application, five outstanding papers out of over 20 were selected for inclusion in this issue of the Chronicle. We feel that the papers presented here will provide interesting and informative reading to all CIF/IFC members and Chronicle subscribers.

The MFC program is exciting and dynamic, designed to meet the many needs of individuals who are interested in specific and essential aspects of forest conservation including fire management systems, forest biomaterials science, forest conservation biology and wildlife ecology, invasive species and threats to forest health, social and cultural ecology of forest ecosystems, sustainable development and economics, and urban forestry. The papers presented in this special issue focus on just some of these topics and themes. Many other past MFC papers covering a wide range of themes are available through the library system at the University of Toronto.

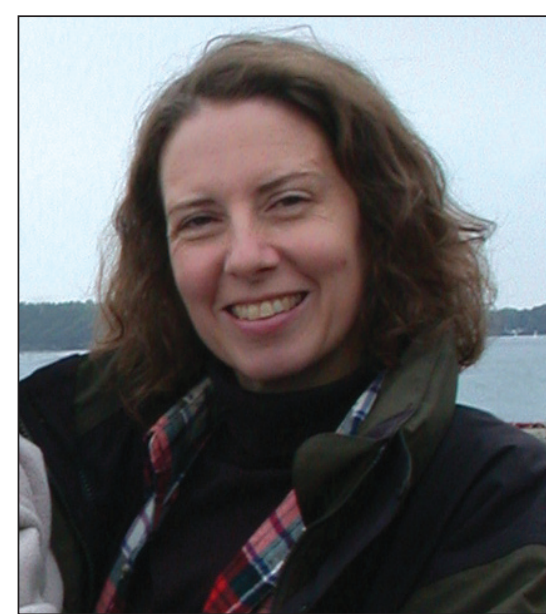

Sandy M. Smith Dean of the Faculty of Forestry University of Toronto Doyenne, Faculté de Foresterie Université de Toronto

On a final note, our Faculty's continuing and mutually beneficial relationship with the Institute, the FRP and the Ontario Ministry of Natural Resources - Southern Science and Information section is one in which we are extremely pleased, and will continue to cultivate in the years ahead. Our annual winter field camp at the Canadian Ecology Centre near Mattawa, Ontario, organized and delivered by these great partners, as well as other helpful groups, is one of a number of highlights for the MFC program. Including a collection of our current cohort's major project papers greatly adds to this important relationship and allows us to continue to provide positive and insightful information to forest professionals and practitioners across Canada.

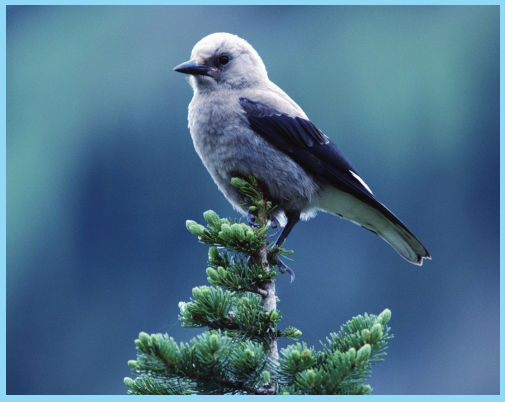

$$
\begin{aligned}
& \text { Invite a callegue to join the CIF/IFC } \\
& \text { Invitey un calligue à se joindse à l'IFC/CIF } \\
& \text { WWW.cif-ifc.org }
\end{aligned}
$$




\section{Publication des textes des diplômés de la Maîtrise en conservation des forêts}

I vec la publication de ces textes pro1 duits par un groupe sélectionné de diplômés de cette année, le Programme de Maîtrise en conservation des forêts (MCF) de la Faculté de Foresterie de l'Université de Toronto se par un groupe sélectionné de Canadian Institute of Forestry/Institut forestier du Canada pour célébrer avec succès la fin de sa $15^{\mathrm{e}}$ année. Depuis ses débuts en 1996, le programme a suscité beaucoup d'intérêt avec plus de 200 diplômés à ce jour, plusieurs à l'œuvre dans les secteurs de la forêt et des ressources naturelles partout au Canada et à l'étranger. Le programme de la MCF a été mis en place par suite de la reconnaissance des valeurs fondamentales, de l'intérêt et des priorités souhaités et attendus par le public canadien et pour mieux faire face la nature étendue et vraiment interdisciplinaire de la foresterie, des sciences forestières, ainsi que de l'aménagement et de la protection des ressources natu-

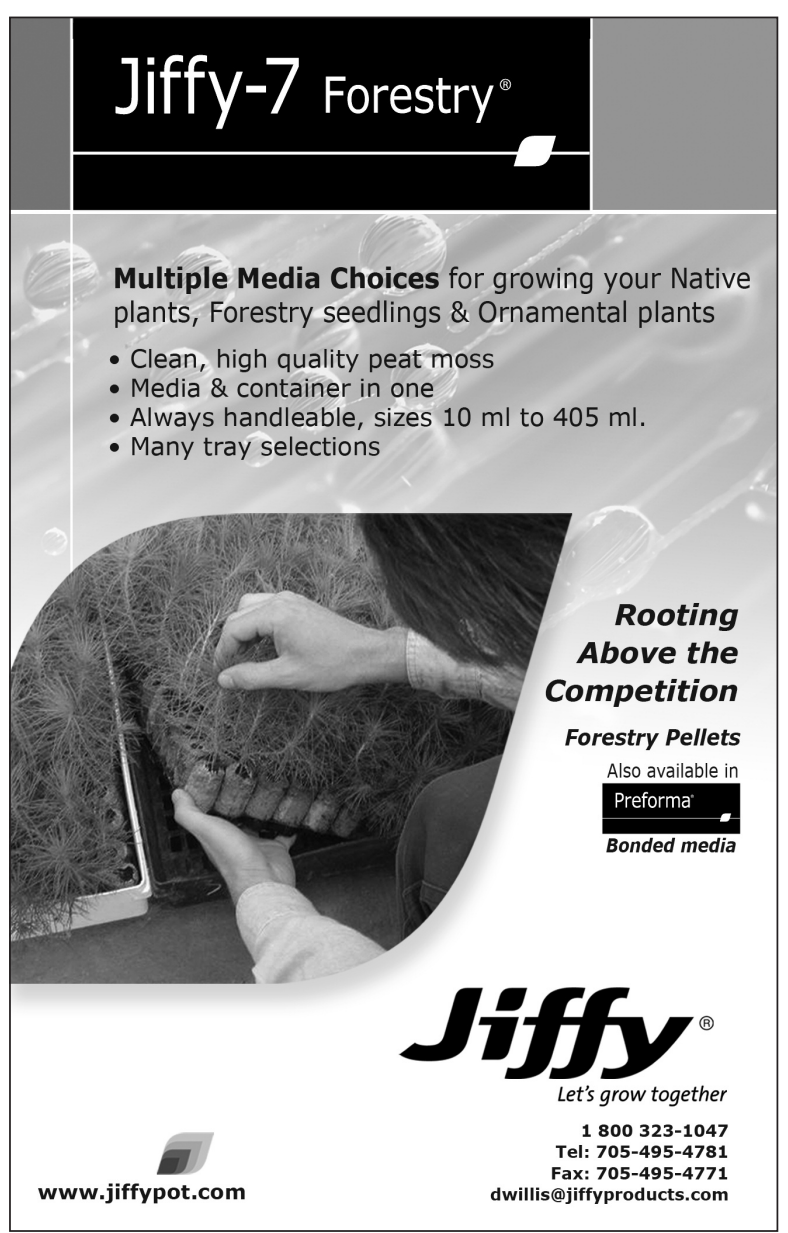

relles. Le programme de la MCF a été conçu pour permettre une formation professionnelle intensive constituée de cours sétalant sur 16 mois en protection des forêts, en plus de travaux pratiques sur le terrain et en laboratoire.

La possibilité de présenter les travaux remarquables de certains de nos étudiants par leur publication dans le Forestry Chronicle est grandement appréciée et constitue, je l'espère, quelque chose qu'il sera possible de poursuivre et qui pourra inciter les autres facultés canadiennes à faire de même. Nos professeurs sont très fiers des recherches entreprises par ces nouveaux professionnels de la foresterie au cours de ce programme et, plus spécifiquement, par les travaux rattachés à leurs mémoires de fin d'études. Au cours des douze mois dédiés aux travaux de recherche et à leur mémoire, chaque étudiant est appuyé par une équipe de conseillers tant de l'interne que de l'externe, ces derniers veillant à assurer la pertinence et l'utilisation pratique des travaux au sein du secteur forestier et des ressources naturelles.

Cette année nous avons eu la chance d'accueillir plusieurs personnes de l'extérieur lors de la présentation des articles en décembre, notamment John Pineau, Directeur général du CIF/ IFC et Matt Meade, Directeur de la formation forestière pour le CIF/IFC et le Partenariat pour la recherche forestière $(\mathrm{PRF})$ et diplômé du programme de la MPF. En fonction de certains critères portant sur la présentation en elle-même, le style de rédaction, le sujet, le thème, la rigueur scientifique, les résultats et la pertinence de l'information et son utilisation, cinq mémoires se sont démarqués parmi les 20 présentations et sont reproduits dans ce numéro du Chronicle. Nous estimons que les mémoires publiés s'avèreront être d'une lecture intéressante et éducative pour tous les membres du CIF/IFC et les abonnés du Forestry Chronicle.

Le programme de la MPF est excitant et dynamique, conçu pour répondre à plusieurs besoins des individus intéressés par les aspects spécifiques et essentiels de la protection des forêts regroupant les systèmes de gestion des feux de forêt, les sciences portant sur les biomatériaux forestiers, les aspects biologiques propres à la protection des forêts et l'écologie de la faune, les espèces envahissantes et les menaces qu'elles posent sur la santé des forêts, lécologie sociale et culturelle des écosystèmes forestiers, le développement durable et le développement économique, ainsi que la foresterie urbaine. Les mémoires publiés dans ce numéro spécial portent sur certains de ces sujets et de ces thèmes. Plusieurs autres mémoires issus de la MPF couvrant un large éventail de sujets sont disponibles par l'entremise du système bibliothécaire de l'Université de Toronto.

Un dernier point, la relation soutenue et mutuellement bénéfique entre la Faculté et l'Institut, le PRF et la division de la Région Sud des Sciences et de l'Information du ministère des Richesses naturelles de l'Ontario constitue une collaboration dont nous sommes très satisfaits et qui continuera à se développer au cours des prochaines années. Notre activité annuelle hivernale au Centre écologique du Canada situé près de Mattawa en Ontario, organisée et réalisée par ces formidables partenaires, ainsi que par d'autres groupes attentionnés, constitue l'un des nombreux faits saillants du programme de la MPF. L'inclusion d'une sélection des mémoires portant sur les travaux principaux de la cohorte d'étudiants de cette année apporte beaucoup à cette importante relation et nous permet de poursuivre la diffusion d'informations positives et significatives aux professionnels et aux praticiens de la foresterie de tout le Canada. 